\title{
Added mass of a pair of disks at small separation
}

\author{
C. ATKINSON ${ }^{1}$ and J. D. SHERWOOD ${ }^{2}$ \\ ${ }^{1}$ Imperial College, Department of Mathematics, Queen's Gate, South Kensington, UK \\ ${ }^{2}$ Department of Applied Mathematics and Theoretical Physics, University of Cambridge, Wilberforce Road, Cambridge \\ CB3 OWA, UK
}

(Received 26 October 2016)

\begin{abstract}
Inviscid irrotational flow around a pair of coaxial disks is considered in the limit in which the distance $2 h$ between the disks is small compared to their radius $a$. The disks have zero thickness and accelerate away from one another along their common axis. The added mass $M$ of each accelerating disk is increased by the presence of the other disk. Analytic predictions are obtained when $h / a \ll 1$, with $M \sim \pi a /(8 h)-\ln (h / a) / 2+0.77875+\cdots$. The term $O(a / h)$ can be obtained by means of an inviscid analysis of approximately unidirectional flow within the gap between the disks, but the correction terms have not been reported previously. The irrotational flow problem satisfies Neumann boundary conditions on the surface of the disks, but is otherwise analogous to the Dirichlet problem of the capacitance of a pair of charged disks, which has been the subject of much study and controversy.
\end{abstract}

\section{Introduction}

The classical problems of the electric field around a disk, or a pair of coaxial disks, have been widely studied: the single disk provides an archetypal problem with mixed boundary conditions, and the pair of disks provides a simple model of a parallel plate capacitor. The problem is usually studied with Dirichlet boundary conditions, with the disks at equal, or equal and opposite, uniform potentials. When the gap $2 h$ between the two disks is small compared to the radius $a$ of the disks, their electrical capacitance may be estimated assuming that there is a uniform electric field between the plates. Edge effects were studied by Maxwell [1], Kirchhoff [2] and Cooke [3] (amongst many), and the leading order edge correction terms to the capacitance are $O(h / a)$ and $O(h / a) \ln (h / a)$. Debate about the magnitude of these terms was eventually settled by Hutson [4], as discussed by Sneddon [5] (section 8.1). Shaw [6] used complex variable methods to determine the edge correction for the electrostatic capacitance, whereas Atkinson \& Leppington [7] used singular perturbation and Wiener-Hopf methods for the case when the two disks are held at equal potential.

Much less attention has been paid to solutions of the Laplace equation with Neumann boundary conditions at the surface of the disks. Here we study such a solution that is of interest in the context of inviscid potential flow. An accelerating body immersed in fluid entrains with it some of the surrounding fluid. The force required to accelerate the body therefore depends not only on the mass of the body itself, but also on the added mass of the accelerating fluid.

The effects of inertia are important in highly transient squeeze-film flows, such as occur in shock absorbers and human joints [8, 9], and interesting hydrodynamic interactions between 
sedimenting disks were observed at Reynolds numbers in the range $80-300$ by Brosse \& Ern $[10,11]$. Here we take the limit of inviscid flow, and consider the acceleration (towards or away from each other) of two coaxial disks immersed in unbounded fluid. The solution that we obtain is expected to be relevant to the case where the two disks move apart from one another, so that fluid flows into the gap between the disks. If the two disks move towards one another, viscous fluid flow will separate from the edges of the disks and emerge as a jet from the gap, rather than adopting the flow field predicted by inviscid potential flow.

Sherwood [12] studied the added mass of a pair of identical, coaxial disks, using physical arguments to obtain leading order asymptotic results for the added mass when the disks were far apart or close together. Results for intermediate separations were found numerically, using Tranter's method [13, 14]. However, the numerical scheme failed when $h \ll a$. There was clearly scope for improved asymptotic analysis when the disks were close together, and our aim here is to provide such analysis. We use methods similar to those of Chen \& Atkinson [15], who looked at the stress and strain fields around a disk-shaped crack in an elastic bimaterial sandwich.

The problem is identical to that of motion of a single disk at depth $h$ towards a horizontal free surface if the surface is not permitted to move vertically. This has been studied in the context of water waves generated by motion of a submerged horizontal disk by Martin \& Farina [16] and by Farina [17]. When the disk oscillates, the real part of the resulting force is equivalent, in the limit of zero frequency, to the added mass studied here. Martin \& Farina [16] expressed their solution in terms of a generalization of Love's integral equation $[18,5]$ that governs the capacitance of a pair of oppositely charged disks, which then had to be solved numerically. Farina [17] considered the limit $h \ll a$, but the resulting approximation for the added mass diverges as the inverse wavenumber $K^{-1}=g / \omega^{2}$, where $\omega$ is the angular frequency of oscillation and $g$ the acceleration due to gravity. Thus this approximation fails in the limit in which we are interested. We note that oppositely charged disks are at (equal and opposite) uniform potentials. Away from the edge of the disks, the electric field between the disks is approximately uniform and normal to the disks across the entire width of the gap. In the problem considered here, the gradient of potential normal to the disks is unifom over the entire surface of each disk, but is equal and opposite on the two disks. Thus the potential for the added mass problem studied here differs markedly from that of the potential around two disks at equal and opposite potential.

\section{Inviscid potential flow around a pair of disks}

We consider inviscid, irrotational flow of incompressible fluid with density $\rho_{l}$. The fluid velocity $\boldsymbol{u}$ can be described in terms of a potential $\phi$ such that $\boldsymbol{u}=\nabla \phi$. Incompressibility then requires

$$
\nabla^{2} \phi=0 .
$$

Consider flow around a rigid body with surface $S_{1}$ and outward facing normal $\boldsymbol{n}$. The centre of the body is at $\boldsymbol{x}_{\mathbf{1}}(t)$ and translates with velocity $\dot{\boldsymbol{x}}=\boldsymbol{U}$, where a dot denotes differentiation with respect to time $t$. The velocity potential around the body, translating at a steady velocity $\boldsymbol{U}$, depends linearly on $\boldsymbol{U}$ and can therefore be written in the form [19]

$$
\phi=\boldsymbol{U}(t) \cdot \boldsymbol{\Phi}\left(\boldsymbol{x}-\boldsymbol{x}_{1}(t)\right),
$$

where the components of $\Phi$ are given by the velocity potentials for motion of the body with unit velocity in the direction of each of the coordinate axes. The force acting on an accelerating 


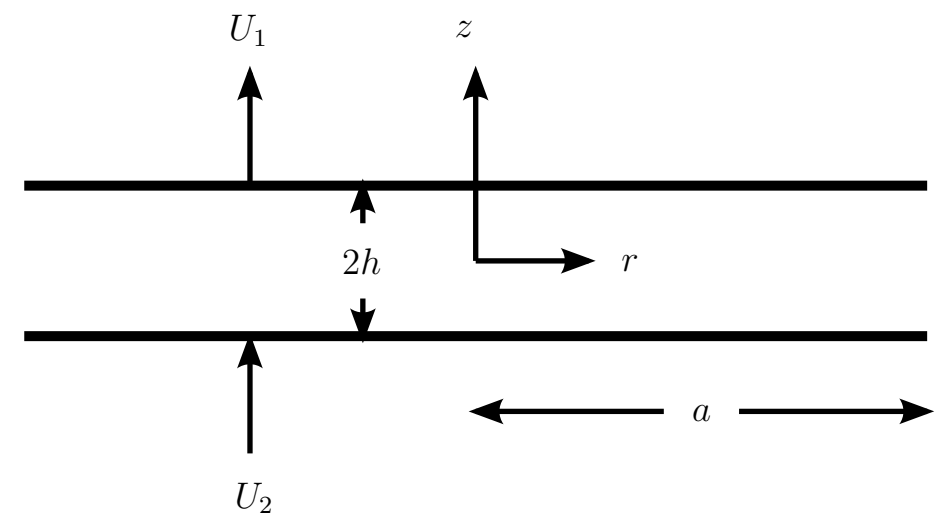

FIGURE 1. The two disks of radius $a$, with separation $2 h \ll a$ on a common axis. We study squeeze flow, in which $U_{2}=-U_{1}$

body may be obtained as an integral of the pressure $p$ over the surface $S$ of the body. Bernoulli's theorem gives the hydrodynamic force $\boldsymbol{F}$ acting on the body:

$$
\boldsymbol{F}=\rho_{l} \frac{\mathrm{d} \boldsymbol{U}}{\mathrm{d} t} \cdot \int_{S_{1}} \boldsymbol{\Phi} \boldsymbol{n} \mathrm{d} S+\rho_{l} \int_{S_{1}}\left[\frac{\boldsymbol{u}^{2}}{2}-\boldsymbol{U} \cdot \boldsymbol{u}\right] \boldsymbol{n} \mathrm{d} S .
$$

Our interest here lies in the first term on the right-hand side of (2.3), which is the acceleration reaction $G$. The second term on the right-hand side of (2.3) ought to represent the force due to steady motion, which is zero in the absence of vorticity [19]. However, this second term is of limited value, since flow of real (i.e. viscous) fluid separates from the disk as it passes its sharp edge, an effect not captured by potential flow theory.

Our aim is to find the reaction force acting on two disks that accelerate away from each other, but we first recall the well known result for the added mass of a single disk of radius $a$ and zero thickness [19]. Consider a disk translating steadily along its axis in the $z$ direction with velocity $U \hat{\boldsymbol{z}}$. We non-dimensionalize all lengths by $a$ and velocities by $U$, and use oblate spheroidal coordinates $(\lambda, \zeta)$ with origin at the centre of the disk, such that $z=\lambda \zeta$ and $r^{2}=$ $\left(1+\lambda^{2}\right)\left(1-\zeta^{2}\right)$. The velocity potential (non-dimensionalized by $U a$ ) in $z>0$ is

$$
\phi=\frac{2}{\pi}\left[z \cot ^{-1}(\lambda)-\zeta\right], \quad(0 \leqslant \zeta \leqslant 1, \quad 0 \leqslant \lambda<\infty) .
$$

The disk $z=0$ corresponds to $\lambda=0$ and thus to $\zeta^{2}=1-r^{2}$, so that $\phi=-2\left(1-r^{2}\right)^{1 / 2} / \pi$ on $z=0^{+}$. The contribution from the upper surface $z=0^{+}$of the disk to the acceleration reaction $G$, nondimensionalized by $a^{3} \rho(\mathrm{d} U / \mathrm{d} t)$, is

$$
\frac{G}{2}=-\frac{2}{\pi} \int_{0}^{1}\left(1-r^{2}\right)^{1 / 2} 2 \pi r \mathrm{~d} r=-\frac{4}{3} \hat{z} .
$$

There is an identical contribution from the lower surface of the disk, and hence the added mass, non-dimensionalized by $\rho a^{3}$, is

$$
M=|\boldsymbol{G}|=8 / 3 .
$$

From now on we consider two coaxial disks, of zero thickness and of radius $a$. The disks are aligned with their axes along the $z$ axis of a cylindrical polar coordinate system $(r, \theta, z)$, with 
disk 1 at $z=h$ and disk 2 at $z=-h$. The disks have velocities $U_{1}(t), U_{2}(t)$ and here we study squeeze flow, for which $U_{2}=-U_{1}$, as depicted in figure 1 .

We non-dimensionalize lengths with respect to $a$, velocities by $U_{1}$, potentials by $a U_{1}$, acceleration reaction by $\rho_{l} a^{3}\left(\mathrm{~d} U_{1} / \mathrm{d} t\right)$ and added mass by $\rho_{l} a^{3}$. The harmonic velocity potential $\phi$ in the half-plane $z \geqslant 0$ has to satisfy

$$
\begin{aligned}
\frac{\partial \phi}{\partial z} & =1, \quad z=h, \quad r<1, \\
\frac{\partial \phi}{\partial z} & =0, \quad z=0, \\
\phi & \rightarrow 0, \quad|\boldsymbol{r}| \rightarrow \infty .
\end{aligned}
$$

The problem is axisymmetric, so that $\phi$ is independent of $\theta$, with $\phi(r, z)=\phi(r,-z)$. The $z$ component of the acceleration reaction on the disk at $z=h$ is

$$
G=\int_{0}^{1} 2 \pi r\left[\phi\left(r, h^{+}\right)-\phi\left(r, h^{-}\right)\right] \mathrm{d} r=-M .
$$

We expect the reaction $G$ to be negative, and the added mass is $M=-G>0$.

We construct the solution for the potential outside the disk in the limit $h \rightarrow 0$ by means of matched asymptotic expansions. First an outer solution, valid far from the disk boundary, is obtained in the form of an integral representation which automatically satisfies the boundary condition $(2.7 b)$ on $z=0$. The condition $(2.7 a)$ on the disk itself is then satisfied approximately as $h \rightarrow 0$, but the solution fails to satisfy an appropriate edge condition on the disk boundary. (A local expansion near the outer edge of the disk shows that we expect $\partial \phi / \partial r \sim(r-a)^{-1 / 2}$ as $r \rightarrow a_{+}$on $z=h$.) This near edge behaviour is accounted for by a suitably scaled inner solution which is matched to the aforementioned outer solution in an appropriate manner. Both outer and inner solution methods will be described below, the outer solution in $\S 3$, the inner solution in $\S 4$, the matching procedure in $\S 5$ and some concluding results in $\S 6$. To help distinguish the outer and inner solutions, we use $V(r, z)$ to denote the outer solution for the potential, and $\phi(X, Z)$ to denote the inner solution.

\section{The outer problem}

\subsection{Integral formulation}

We use the integral representation of an axisymmetric harmonic function $[5,20]$, adapted to satisfy the boundary condition $(2.7 b)$

$$
V(r, z)=\operatorname{Im}\left\{\int_{0}^{1}\left[\frac{g(t)}{\left[r^{2}+(h-z-\mathrm{i} t)^{2}\right]^{1 / 2}}-\frac{g(t)}{\left[r^{2}+(h+\mathrm{i} t+z)^{2}\right]^{1 / 2}}\right] \mathrm{d} t\right\},
$$

where $g(t)$ is a real continuous function. The square roots in (3.1) are defined in terms of $u=$ $h \mp z$ by

$$
\begin{gathered}
{\left[r^{2}+(u+\mathrm{i} t)^{2}\right]^{1 / 2}=s \exp (\mathrm{i} \psi / 2), \quad(s \geqslant 0)} \\
s^{2} \cos \psi=r^{2}+u^{2}-t^{2} \quad, \quad s^{2} \sin \psi=2 u t, \quad(-\pi \leqslant \psi \leqslant \pi) \\
{\left[r^{2}+(u-\mathrm{i} t)^{2}\right]^{1 / 2}=s \exp (-\mathrm{i} \psi / 2) .}
\end{gathered}
$$


We solve the problem in the half space $z \geqslant 0$, so that when $u>0$ (as is always the case for $u=z+h$ ) then $0 \leqslant \psi \leqslant \pi$. However, on $z=h$ the first term in (3.1) has a jump as $u=h-z$ goes from positive to negative since

$$
\begin{aligned}
& \lim _{u \rightarrow 0_{-}^{+}}\left[r^{2}+(u+\mathrm{i} t)^{2}\right]^{1 / 2}=\left(r^{2}-t^{2}\right)^{1 / 2}, \quad r^{2}>t^{2} \\
& \lim _{u \rightarrow 0_{-}^{+}}\left[r^{2}+(u+\mathrm{i} t)^{2}\right]^{1 / 2}= \pm i\left(t^{2}-r^{2}\right)^{1 / 2}, \quad t^{2}>r^{2} .
\end{aligned}
$$

Hence on $z=h$ we have the jump in potential

$$
[V(r, z)]_{z=h_{-}}^{z=h_{+}}=-2 \int_{r}^{1} \frac{g(t) \mathrm{d} t}{\left(t^{2}-r^{2}\right)^{1 / 2}}
$$

which is the jump in potential that contributes to the integral (2.8) for the added mass.

The gradient of the potential is

$$
\frac{\partial V}{\partial z}=\operatorname{Im}\left\{\int_{0}^{1} g(t)\left[\frac{(h-z-\mathrm{i} t)}{\left[r^{2}+(h-z-\mathrm{i} t)^{2}\right]^{3 / 2}}+\frac{(h+z+\mathrm{i} t)}{\left[r^{2}+(h+z+\mathrm{i} t)^{2}\right]^{3 / 2}}\right] \mathrm{d} t\right\} .
$$

When $z=0$, the integrand in (3.5) is real, so that $\partial V / \partial z=0$ is automatically satisfied. We now have to choose $g(t)$ such that

$$
\frac{\partial V}{\partial z}=1, \quad z=h, \quad r<1 .
$$

To avoid singular behaviour in the integrands of Eq. (3.5) when applying the boundary condition (3.6) in the limit $z \rightarrow h$, we replace this boundary condition by one obtained by repeated integration of (3.5) and (3.6) with respect to a new variable $r_{1}=r^{2}$. Integration of (3.5) gives

$$
\begin{aligned}
Q(r, z) & =\int_{0}^{r} r^{\prime} \frac{\partial V}{\partial z} \mathrm{~d} r^{\prime} \\
& =-\operatorname{Im}\left\{\int_{0}^{1}\left[\frac{g(t)(h-z-\mathrm{i} t)}{\left[r^{2}+(h-z-\mathrm{i} t)^{2}\right]^{1 / 2}}+\frac{g(t)(h+z+\mathrm{i} t)}{\left[r^{2}+(h+\mathrm{i} t+z)^{2}\right]^{1 / 2}}-2 g(t)\right] \mathrm{d} t\right\},
\end{aligned}
$$

where the last term on the right-hand side is zero since $g(t)$ is real. Hence

$$
\begin{gathered}
P(r, z)=\int_{0}^{r} r^{\prime} Q\left(r^{\prime}, z\right) \mathrm{d} r^{\prime}=-\operatorname{Im}\left\{\int_{0}^{1} g(t)(h-z-\mathrm{i} t)\left[r^{2}+(h-z-\mathrm{i} t)^{2}\right]^{1 / 2} \mathrm{~d} t\right. \\
+\int_{0}^{1} g(t)(h+z+\mathrm{i} t)\left[r^{2}+(h+\mathrm{i} t+z)^{2}\right]^{1 / 2} \mathrm{~d} t \\
\left.-\int_{0}^{1} g(t)\left[(h-z-\mathrm{i} t)^{2}+(h+z+\mathrm{i} t)^{2}\right] \mathrm{d} t\right\}
\end{gathered}
$$

and

$$
\begin{aligned}
R(r, z) & =\int_{0}^{r} r^{\prime} P\left(r^{\prime}, z\right) \mathrm{d} r^{\prime}=-\operatorname{Im}\left\{\frac{1}{3}\left[\int_{0}^{1} g(t)(h-z-\mathrm{i} t)\left[r^{\prime 2}+(h-z-\mathrm{i} t)^{2}\right]^{3 / 2} \mathrm{~d} t\right]_{r^{\prime}=0}^{r}\right. \\
& \left.+\frac{1}{3}\left[\int_{0}^{1} g(t)(h+z+\mathrm{i} t)\left[r^{\prime 2}+(h+\mathrm{i} t+z)^{2}\right]^{3 / 2} \mathrm{~d} t\right]_{r^{\prime}=0}^{r}-2 z r^{2} \mathrm{i} \int_{0}^{1} g(t) t \mathrm{~d} t\right\} .
\end{aligned}
$$


The boundary condition (3.6) implies that on $z=h$ the left-hand sides of (3.7), (3.8) and (3.9) are

$$
Q(r, h)=\frac{r^{2}}{2} \quad, \quad P(r, h)=\frac{r^{4}}{8} \quad, \quad R(r, h)=\frac{r^{6}}{48} \quad, \quad r<1 .
$$

To evaluate the right-hand side of (3.9) in the limit $h \rightarrow z$, we require certain limits and expansions of the square roots, e.g.

$$
\begin{aligned}
\lim _{z \rightarrow h}\left[r^{2}+(h-z-\mathrm{i} t)^{2}\right]^{3 / 2} & =\left(r^{2}-t^{2}\right)^{3 / 2}, \quad r \geqslant t, \\
& =\mathrm{i}\left(t^{2}-r^{2}\right)^{3 / 2}, \quad r \leqslant t,
\end{aligned}
$$

and

$$
\begin{aligned}
\lim _{z \rightarrow h}\left[r^{2}+(h+z+\mathrm{i} t)^{2}\right]^{3 / 2} & =\left[r^{2}-t^{2}+4 h^{2}+4 h t \mathrm{i}\right]^{3 / 2} \\
& =\left(r^{2}-t^{2}\right)^{3 / 2}\left[1+\frac{6 h t \mathrm{i}}{r^{2}-t^{2}}+\frac{6 h^{2}\left(r^{2}-2 t^{2}\right)}{\left(r^{2}-t^{2}\right)^{2}}+O\left(h^{3}\right)\right] \quad r>t, \\
& =-\mathrm{i}\left(t^{2}-r^{2}\right)^{3 / 2}\left[1+\frac{6 h t \mathrm{i}}{r^{2}-t^{2}}+\frac{6 h^{2}\left(r^{2}-2 t^{2}\right)}{\left(r^{2}-t^{2}\right)^{2}}+O\left(h^{3}\right)\right], \quad r \leqslant t .
\end{aligned}
$$

We note that these expansions fail in a region $|r-t|=O(h)$. This region makes a contribution $O\left(g h^{5 / 2}\right)$ to the integrals in (3.9), and may be neglected at the order to which we shall work. When the expansions (3.12) are inserted into the right-hand side of (3.9), all terms up to $O\left(h^{2}\right)$ are integrable: had we desired to continue to higher order in $h$, further integrations with respect to $r^{\prime}$ of the type used to go from (3.7) to (3.8) to (3.9) would be necessary.

Thus, taking the limit $z \rightarrow h$ in (3.9), and expanding the right-hand side in powers of $h$, we obtain

$$
\begin{aligned}
\frac{r^{6}}{48} & =h \int_{r}^{1} g(t)\left(t^{2}-r^{2}\right)^{3 / 2}\left[\frac{2}{3}-\frac{2 t^{2}}{r^{2}-t^{2}}\right] \mathrm{d} t+h \int_{0}^{1} g(t)\left[2 r^{2} t-\frac{8 t^{3}}{3}\right] \mathrm{d} t \\
& -2 h^{2} \int_{0}^{r} g(t) t\left(r^{2}-t^{2}\right)^{3 / 2} \frac{\left[3 r^{2}-4 t^{2}\right]}{\left(r^{2}-t^{2}\right)^{2}} \mathrm{~d} t+O\left(h^{3}\right) .
\end{aligned}
$$

Differentiating both sides of (3.13) with respect to $r$ gives

$$
\begin{aligned}
\frac{r^{5}}{8} & =-2 h^{2} r^{2} g(0)-2 r h^{2} \int_{0}^{r} g^{\prime}(t) \frac{\left(r^{2}-2 t^{2}\right)}{\left(r^{2}-t^{2}\right)^{1 / 2}} \mathrm{~d} t \\
& -2 h r \int_{r}^{1} g(t)\left(t^{2}-r^{2}\right)^{1 / 2} \mathrm{~d} t-2 h r \int_{r}^{1} \frac{g(t) t^{2} \mathrm{~d} t}{\left(t^{2}-r^{2}\right)^{1 / 2}}+4 r h \int_{0}^{1} g(t) t \mathrm{~d} t .
\end{aligned}
$$

We expand $g(t)$ as

$$
g=g^{(0)}(t)+h g^{(1)}(t)+\cdots
$$

so that at $O(h)$

$$
\frac{r^{4}}{8}=-2 h \int_{r}^{1} g^{(0)}(t)\left(t^{2}-r^{2}\right)^{1 / 2} \mathrm{~d} t-2 h \int_{r}^{1} \frac{g^{(0)}(t) t^{2} \mathrm{~d} t}{\left(t^{2}-r^{2}\right)^{1 / 2}}+4 h \int_{0}^{1} g^{(0)}(t) t \mathrm{~d} t,
$$


and at $O\left(h^{2}\right)$

$$
\begin{aligned}
0 & =-r g^{(0)}(0)-\int_{0}^{r} g^{(0)^{\prime}}(t) \frac{\left(r^{2}-2 t^{2}\right)}{\left(r^{2}-t^{2}\right)^{1 / 2}} \mathrm{~d} t \\
& -\int_{r}^{1} g^{(1)}(t)\left(t^{2}-r^{2}\right)^{1 / 2} \mathrm{~d} t-\int_{r}^{1} \frac{g^{(1)}(t) t^{2} \mathrm{~d} t}{\left(t^{2}-r^{2}\right)^{1 / 2}}+2 \int_{0}^{1} g^{(1)}(t) t \mathrm{~d} t .
\end{aligned}
$$

To solve (3.14) we set

$$
t_{1}=t^{2} \quad, \quad r_{1}=r^{2} \quad, \quad g_{i}\left(t_{1}\right)=\frac{h g^{(i)}(t)}{t}, \quad i=0,1, \ldots
$$

so that the expansion (3.15) becomes

$$
g=\frac{g_{0}\left(t_{1}\right)}{h t}+\frac{g_{1}\left(t_{1}\right)}{t}+\cdots
$$

The leading order equation (3.16) becomes

$$
\frac{r_{1}^{2}}{8}=-\int_{r_{1}}^{1} g_{0}\left(t_{1}\right)\left[\left(t_{1}-r_{1}\right)^{1 / 2}+\frac{t_{1}}{\left(t_{1}-r_{1}\right)^{1 / 2}}\right] \mathrm{d} t_{1}+2 \int_{0}^{1} t_{1}^{1 / 2} g_{0}\left(t_{1}\right) \mathrm{d} t_{1} .
$$

We now multiply (3.20) by $\left(r_{1}-\bar{r}_{1}\right)^{-1 / 2}$ and integrate over $r_{1}$ from $r_{1}=\bar{r}_{1}$ to $r_{1}=1$ to obtain

$$
\begin{gathered}
0=-\frac{1}{60}\left(8 \bar{r}_{1}^{2}+4 \bar{r}_{1}+3\right)\left(1-\bar{r}_{1}\right)^{1 / 2}+4\left(1-\bar{r}_{1}\right)^{1 / 2} \int_{0}^{1} t_{1}^{1 / 2} g_{0}\left(t_{1}\right) \mathrm{d} t_{1} \\
-\int_{\bar{r}_{1}}^{1} \frac{\mathrm{d} r_{1}}{\left(r_{1}-\bar{r}_{1}\right)^{1 / 2}} \int_{r_{1}}^{1} g_{0}\left(t_{1}\right)\left[\left(t_{1}-r_{1}\right)^{1 / 2}+\frac{t_{1}}{\left(t_{1}-r_{1}\right)^{1 / 2}}\right] \mathrm{d} t_{1} .
\end{gathered}
$$

But if we change the integration variable $r_{1}$ in (3.21) by setting $r_{1}-\bar{r}_{1}=\left(t_{1}-\bar{r}_{1}\right) \sin ^{2} \theta$ and interchange the order of integration with respect to $\theta$ and $t_{1},(3.21)$ becomes

$$
\begin{aligned}
\pi \int_{\bar{r}_{1}}^{1} g_{0}\left(t_{1}\right)\left[\frac{\left(t_{1}-\bar{r}_{1}\right)}{2}+t_{1}\right] \mathrm{d} t_{1} & =-\frac{1}{60}\left(8 \bar{r}_{1}^{2}+4 \bar{r}_{1}+3\right)\left(1-\bar{r}_{1}\right)^{1 / 2} \\
& +4\left(1-\bar{r}_{1}\right)^{1 / 2} \int_{0}^{1} t_{1}^{1 / 2} g_{0}\left(t_{1}\right) \mathrm{d} t_{1}
\end{aligned}
$$

We now differentiate (3.22) twice with respect to $\bar{r}_{1}$ to obtain

$$
\begin{aligned}
\pi\left[g_{0}\left(\bar{r}_{1}\right)+\bar{r}_{1} g_{0}{ }^{\prime}\left(\bar{r}_{1}\right)-\frac{g_{0}\left(\bar{r}_{1}\right)}{2}\right] & =\frac{4}{15}\left(1-\bar{r}_{1}\right)^{1 / 2}-\frac{4 \bar{r}_{1}+1}{15\left(1-\bar{r}_{1}\right)^{1 / 2}}-\frac{8 \bar{r}_{1}^{2}+4 \bar{r}_{1}+3}{240\left(1-\bar{r}_{1}\right)^{3 / 2}} \\
& +\frac{1}{\left(1-\bar{r}_{1}\right)^{3 / 2}} \int_{0}^{1} t_{1}^{1 / 2} g_{0}\left(t_{1}\right) \mathrm{d} t_{1}
\end{aligned}
$$

i.e.

$$
\pi \frac{\mathrm{d}}{\mathrm{d} \bar{r}_{1}}\left[\bar{r}_{1}^{1 / 2} g_{0}\left(\bar{r}_{1}\right)\right]=\frac{\left(8 \bar{r}_{1}^{2}-12 \bar{r}_{1}+3\right) \bar{r}_{1}^{-1 / 2}}{16\left(1-\bar{r}_{1}\right)^{3 / 2}}-\frac{\bar{r}_{1}^{-1 / 2}}{\left(1-\bar{r}_{1}\right)^{3 / 2}} \int_{0}^{1} t_{1}^{1 / 2} g_{0}\left(t_{1}\right) \mathrm{d} t_{1},
$$

which integrates to give

$$
g^{(0)}(t)=\frac{t}{h} g_{0}\left(t_{1}\right)=\frac{3 t-4 t^{3}}{8 h \pi\left(1-t^{2}\right)^{1 / 2}}+\frac{2 I t}{\pi h\left(1-t^{2}\right)^{1 / 2}},
$$


where

$$
I=\int_{0}^{1} t_{1}^{1 / 2} g_{0}\left(t_{1}\right) \mathrm{d} t_{1}
$$

is as yet unknown. The final term on the right-hand side of (3.25), involving $I$, can be shown by substitution to be an eigensolution of the original integral equation (3.22).

The jump in $V$ (3.1) across the plate is

$$
V_{h^{+}}-V_{h^{-}}=2 \operatorname{Im}\left\{\int_{r}^{1}\left[\frac{g(t)}{\mathrm{i}\left(t^{2}-r^{2}\right)^{1 / 2}}\right] \mathrm{d} t\right\}=-2 \int_{r}^{1}\left[\frac{g^{(0)}(t)+h g^{(1)}(t)+\cdots}{\left(t^{2}-r^{2}\right)^{1 / 2}}\right] \mathrm{d} t
$$

where $V_{h^{ \pm}}=V\left(r, z=h^{ \pm}\right)$. If we insert equation (3.25) for $g^{(0)}$ into (3.27), we find at leading order

$$
V_{h^{+}}^{(0)}-V_{h^{-}}^{(0)}=-\frac{1-2 r^{2}}{8 h}-\frac{2 I}{h} .
$$

But we require $V_{h^{+}}-V_{h^{-}}=0$ at $r=1$, so that, by (3.28), $I=1 / 16$ and hence

$$
V_{h^{+}}^{(0)}-V_{h^{-}}^{(0)}=\frac{r^{2}-1}{4 h}
$$

and, by (3.25),

$$
g^{(0)}(t)=\frac{t\left(1-t^{2}\right)^{1 / 2}}{2 \pi h} .
$$

The solution of the $O\left(h^{2}\right)$ equation (3.17), in which $g^{(0)}$ appears as a forcing term, may be found by the same method used to obtain the solution (3.25) of the $O(h)$ equation (3.16). We find

$$
h g^{(1)}(t)=\frac{\left(1-2 t^{2}\right)}{2 \pi^{2}\left(1-t^{2}\right)^{1 / 2}} \ln \left[\frac{1+t}{1-t}\right]-\frac{A t}{\pi\left(1-t^{2}\right)^{1 / 2}},
$$

where $A$ is as yet unknown, and the final term on the right-hand side of (3.31), involving $A$, is again an eigensolution of the integral equation. The contribution at second order to the jump in potential across the disk is, by (3.27),

$$
\begin{aligned}
{\left[V^{(1)}\right]_{z=h} } & =V_{h^{+}}^{(1)}-V_{h^{-}}^{(1)}=-2 \int_{r}^{1} \frac{h g^{(1)}(t) \mathrm{d} t}{\left(t^{2}-r^{2}\right)^{1 / 2}}=-\int_{r_{1}}^{1} \frac{g_{1}\left(t_{1}\right) \mathrm{d} t_{1}}{\left(t_{1}-r_{1}\right)^{1 / 2}} \\
& =A-\int_{r_{1}}^{1} \frac{\left(1-2 t_{1}\right)}{2 \pi^{2} t_{1}^{1 / 2}\left(1-t_{1}\right)^{1 / 2}\left(t_{1}-r_{1}\right)^{1 / 2}} \ln \left[\frac{1+t_{1}^{1 / 2}}{1-t_{1}^{1 / 2}}\right] \mathrm{d} t_{1},
\end{aligned}
$$

where we recall that $r_{1}=r^{2}$.

At $O\left(h^{2}\right)$, no choice of the undetermined constant $A$ allows the condition $[V]=0$ to be met at $r=1$. This is a consequence of the singular nature of the problem. The outer solution cannot satisfy conditions at $r=1$, but we expect it to match asymptotically with the inner solution, to be obtained in the next section, which does indeed satisfy the condition $[V]=0$ at $r=1$.

\section{The inner problem}

Here we scale the problem so that the neighbourhood of the edge at $r=1$ is emphasized and as a consequence in the limit $h \rightarrow 0$ the boundary condition $(2.7 a)$ on the plate is replaced by a condition that the normal gradient becomes zero, see $(4.5 a)$ below. We shall solve the inner problem by means of a Wiener-Hopf analysis. This inner problem has eigensolutions which are 
determined by matching at infinity (in the inner coordinate system) to the outer solutions once the latter have been rewritten in terms of the inner coordinates.

\subsection{Zeroth order solution of the Laplace equation}

In order to study the potential near the outer edge of the gap between the disks in the limit $h=0$ (when the gap vanishes), we define inner coordinates $(X, Z)$ such that

$$
r=1+h X \quad, \quad z=h Z,
$$

and when $h \rightarrow 0$ we look for an expansion

$$
\phi^{(\mathrm{in})}=\phi^{(0)}+h \phi^{(1)}+\cdots .
$$

The inner solutions then satisfy

$$
\begin{gathered}
\frac{\partial^{2} \phi^{(0)}}{\partial X^{2}}+\frac{\partial^{2} \phi^{(0)}}{\partial Z^{2}}=0, \\
\frac{\partial^{2} \phi^{(1)}}{\partial X^{2}}+\frac{\partial^{2} \phi^{(1)}}{\partial Z^{2}}+\frac{\partial \phi^{(0)}}{\partial X}=0,
\end{gathered}
$$

with boundary conditions (2.7) becoming, in the limit $h \rightarrow 0$,

$$
\begin{aligned}
& \frac{\partial \phi^{(\mathrm{in})}}{\partial Z}=h, \quad \text { on } Z=1 \text { for } X<0, \\
& \frac{\partial \phi^{(\mathrm{in})}}{\partial Z}=0, \quad \text { on } Z=0, \forall X .
\end{aligned}
$$

We now look for a solution to the inner problem in the limit $h \rightarrow 0$, and take the Fourier transform

$$
\bar{\phi}=\int_{-\infty}^{\infty} \mathrm{e}^{\mathrm{i} \xi X} \phi(x, z) \mathrm{d} x .
$$

The leading order inner solution satisfies (4.3), which in Fourier transform space is

$$
\frac{\mathrm{d}^{2} \bar{\phi}_{0}}{\mathrm{~d} Z^{2}}-\xi^{2} \bar{\phi}_{0}=0
$$

We look for a solution of (4.7) of the form

$$
\begin{aligned}
\bar{\phi}_{0} & =\bar{\Phi}_{1}^{(0)}(\xi, Z)=B_{1} \cosh (|\xi| Z), \quad Z<1, \\
& =\bar{\Phi}_{2}^{(0)}(\xi, Z)=B_{2} \exp (-|\xi| Z), \quad Z>1 .
\end{aligned}
$$

Continuity of $\partial \bar{\phi} / \partial z$ on $Z=1$ requires

$$
-B_{2} \exp (-|\xi|)=B_{1} \sinh (|\xi|) .
$$

The potential $\phi$ is continuous for all $X>0$ on $Z=1$, so that on $Z=1$

$$
\bar{\Phi}_{1}^{(0)}\left(\xi, 1^{-}\right)-\bar{\Phi}_{2}^{(0)}\left(\xi, 1^{+}\right)=B_{1} \cosh (|\xi|)-B_{2} \exp (-|\xi|) \equiv U_{-}^{(0)},
$$

where we use a subscript - to indicate that a function is analytic in some region $\Im(\xi)<\epsilon$ of the complex plane, whereas a subscript + indicates a function analytic in $\Im(\xi)>\epsilon$. Using (4.9), we can write (4.10) as

$$
U_{-}^{(0)}=-B_{2}[\operatorname{coth}(|\xi|)+1] \exp (-|\xi|)=B_{1} \exp (|\xi|) .
$$


Moreover, on $Z=1$

$$
\frac{\partial \bar{\Phi}_{2}^{(0)}}{\partial Z}=-|\xi| B_{2} \exp (-|\xi|)=W_{+}^{(0)},
$$

and so eliminating $B_{2}$ from (4.11) and (4.12) gives

$$
U_{-}^{(0)}=\frac{W_{+}^{(0)}}{|\xi|}[\operatorname{coth}(|\xi|)+1]=\frac{W_{+}^{(0)}}{G(\xi)}
$$

where

$$
G(\xi)=|\xi| \exp (-|\xi|) \sinh (|\xi|) .
$$

The next step is to factorise the function $G(\xi)$ into the product of plus and minus functions,

$$
G(\xi)=G_{+}(\xi) G_{-}(\xi)
$$

where

$$
\begin{aligned}
& G_{+}(\xi)=\frac{1}{\Gamma(1-\mathrm{i} \xi / \pi)} \exp \left[-\frac{\mathrm{i} \xi}{\pi} \ln \left(-\frac{\mathrm{i} \xi}{\pi}\right)\right] \mathrm{e}^{\mathrm{i} \xi / \pi} \xi, \\
& G_{-}(\xi)=\frac{1}{\Gamma(1+\mathrm{i} \xi / \pi)} \exp \left[\frac{\mathrm{i} \xi}{\pi} \ln \left(\frac{\mathrm{i} \xi}{\pi}\right)\right] \mathrm{e}^{-\mathrm{i} \xi / \pi} \xi,
\end{aligned}
$$

where $\Gamma$ is the Gamma function. This factorisation (4.16) makes use of the factorisation

$$
\exp (-|\xi|)=\exp \left[\frac{\mathrm{i} \xi}{\pi} \ln \left(\frac{\mathrm{i} \xi}{\pi}\right)\right] \exp \left[-\frac{\mathrm{i} \xi}{\pi} \ln \left(-\frac{\mathrm{i} \xi}{\pi}\right)\right]
$$

where $\ln (\mathrm{i} \xi / \pi)$ is analytic on the complex plane with a cut from $\mathrm{i} \epsilon$ to $\mathrm{i} \infty, \ln (-\mathrm{i} \xi / \pi)$ is analytic on the complex plane with a cut from $-\mathrm{i} \epsilon$ to $-\mathrm{i} \infty$, and we let $\epsilon$ tend to zero. The $\Gamma$ functions in (4.16) follow from the factorisation $|\xi| / \sinh (|\xi|)=\Gamma(1-\mathrm{i} \xi / \pi) \Gamma(1+\mathrm{i} \xi / \pi)$. In addition, one can show from the behaviour of the $\Gamma$ function as $|\xi| \rightarrow \infty$ that

$$
G_{+}(\xi) \sim \xi_{+}^{1 / 2} \quad, \quad G_{-}(\xi) \sim \xi_{-}^{1 / 2} \quad, \quad \frac{1}{G_{-}} \sim\left(\frac{2 \mathrm{i}}{\xi_{-}}\right)^{1 / 2}\left[1+\frac{\pi}{12 \mathrm{i} \xi}+\cdots\right],
$$

in respective half planes, where $\xi_{+}^{1 / 2}$ is analytic in the complex plane cut from $-\mathrm{i} \epsilon$ to $-\infty$ and $\xi_{-}^{1 / 2}$ is analytic in the complex plane cut from $i \epsilon$ to $\infty$.

Equation (4.13) can now be rearranged as

$$
U_{-}^{(0)} G_{-}=\frac{W_{+}^{(0)}}{G_{+}}
$$

But on $Z=1$ we expect the solution of the Laplace equation at the edge of the disk to have the same square root singularity as on an isolated disk. Hence $\phi \sim X^{1 / 2}$ and $\partial \phi / \partial X \sim X^{-1 / 2}$ as $X \rightarrow 0$. We therefore expect $U_{-} \sim \xi^{-3 / 2}$ and $W \sim \xi^{-1 / 2}$ as $\xi \rightarrow \infty$, and so

$$
\frac{W_{+}^{(0)}}{G_{+}}=U_{-}^{(0)} G_{-}=\frac{\hat{A}}{\xi} \text {. }
$$

To expand $G_{-}(4.16 b)$ for $\xi \ll 1$, we note that

$$
\Gamma(1+\mathrm{i} \xi / \pi)=1+\frac{\mathrm{i} \xi}{\pi} \Gamma^{\prime}(1)+\cdots=1-\frac{\mathrm{i} \xi \gamma}{\pi}+\cdots,
$$


where $\Gamma^{\prime}(1)=-\gamma=-0.57721 \ldots$, and

$$
\exp \left[\frac{\mathrm{i} \xi}{\pi} \ln \left(\frac{\mathrm{i} \xi}{\pi}\right)-\frac{\mathrm{i} \xi}{\pi}\right]=1-\frac{\mathrm{i} \xi}{\pi}+\frac{\mathrm{i} \xi}{\pi} \ln \left(\frac{\mathrm{i} \xi}{\pi}\right)+\cdots,
$$

so that

$$
G_{-} \sim\left(1-\frac{\mathrm{i} \xi \gamma}{\pi}+\cdots\right)^{-1}\left(1-\frac{\mathrm{i} \xi}{\pi}+\frac{\mathrm{i} \xi}{\pi} \ln \left(\frac{\mathrm{i} \xi}{\pi}\right)+\cdots\right) \xi
$$

and hence

$$
G_{-}^{-1} \sim \frac{1}{\xi}\left(1+\frac{\mathrm{i} \xi}{\pi}\left[(1-\gamma)-\ln \left(\frac{\mathrm{i} \xi}{\pi}\right)\right]-\frac{\xi^{2} \gamma}{\pi^{2}} \ln \left(\frac{\mathrm{i} \xi}{\pi}\right)+\cdots\right) .
$$

Hence, inserting (4.16b) for $G_{-}$into (4.20) we find that in the limit $\xi \rightarrow 0$,

$$
U_{0}^{-}=\frac{\hat{A}}{\xi G_{-}} \approx \hat{A}\left[\frac{1}{\xi^{2}}-\frac{\mathrm{i}}{\xi \pi}(\gamma+\ln (\mathrm{i} \xi))+\frac{\mathrm{i}}{\xi \pi}(\ln \pi+1)\right] \quad \text { as } \xi \rightarrow 0 .
$$

We find the asymptotic behaviour of $\left[V^{(0)}\right]=V_{h^{+}}^{(0)}-V_{h^{-}}^{(0)}$ in the limit $X \rightarrow-\infty$ by inverting $U_{0}^{-}=\bar{\Phi}_{1}^{(0)}\left(\xi, 1^{-}\right)-\bar{\Phi}_{2}^{(0)}\left(\xi, 1^{+}\right)$in the limit $\xi \rightarrow 0$. To perform the inversion of (4.25) we note the transforms

$$
\begin{gathered}
\int_{-\infty}^{0} \mathrm{e}^{\mathrm{i} \xi X} \mathrm{~d} X=-\frac{\mathrm{i}}{\xi}, \quad \int_{-\infty}^{0} X \mathrm{e}^{\mathrm{i} \xi X} \mathrm{~d} X=\frac{1}{\xi^{2}}, \quad \int_{-\infty}^{0} X^{2} \mathrm{e}^{\mathrm{i} \xi X} \mathrm{~d} X=\frac{2 \mathrm{i}}{\xi^{3}}, \\
\int_{-\infty}^{0} \ln (|X|) \mathrm{e}^{\mathrm{i} \xi X} \mathrm{~d} X=\frac{\mathrm{i}}{\xi}(\gamma+\ln \xi), \quad \int_{-\infty}^{0} X \ln (|X|) \mathrm{e}^{\mathrm{i} \xi X} \mathrm{~d} X=\frac{1-\gamma}{\xi^{2}}-\frac{\ln \xi}{\xi^{2}},
\end{gathered}
$$

which imply

$$
\left[V^{(0)}\right]=V_{h^{+}}^{(0)}-V_{h^{-}}^{(0)}=-\hat{A}\left\{X-\frac{1+\ln \pi}{\pi}-\frac{\ln (-X)}{\pi}\right\}
$$

Similarly, to find the asymptotic behaviour of $\left[V^{(0)}\right]=V_{h^{+}}^{(0)}-V_{h^{-}}^{(0)}$ in the limit $X \rightarrow 0$ we invert $U_{0}^{-}=\bar{\Phi}_{1}^{(0)}\left(\xi, 1^{-}\right)-\bar{\Phi}_{2}^{(0)}\left(\xi, 1^{+}\right)$in the limit $\xi \rightarrow \infty$ using the transforms

$$
\int_{-\infty}^{0}(-X)^{1 / 2} \mathrm{e}^{\mathrm{i} \xi X} \mathrm{~d} X=\frac{\pi^{1 / 2}}{2(\mathrm{i} \xi)^{3 / 2}}, \quad-\mathrm{i} \int_{-\infty}^{0}(-X)^{3 / 2} \mathrm{e}^{\mathrm{i} \xi X} \mathrm{~d} X=-\frac{3 \pi^{1 / 2}}{4 \mathrm{i}^{3 / 2} \xi^{5 / 2}} .
$$

By (4.18) and (4.20),

$$
U_{-}^{(0)} \sim \frac{\hat{A}(2 i)^{1 / 2}}{\xi^{3 / 2}}\left[1+\frac{\pi}{12 \mathrm{i} \xi}\right] \quad \text { as } \xi \rightarrow \infty
$$

which inverts to give

$$
\left[V^{(0)}\right]=V_{h^{+}}^{(0)}-V_{h^{-}}^{(0)}=2 \hat{A}\left(\frac{2}{\pi}\right)^{1 / 2}(-X)^{1 / 2}+\frac{\hat{A}}{9}(2 \pi)^{1 / 2}(-X)^{3 / 2} \quad \text { as } X \rightarrow 0 .
$$

\subsection{First order inner problem}

We take Fourier transforms of (4.4), to obtain

$$
\frac{\mathrm{d}^{2} \bar{\phi}^{(1)}}{\mathrm{d} Z^{2}}-\xi^{2} \bar{\phi}^{(1)}-\mathrm{i} \xi \bar{\phi}^{(0)}=0
$$


with solution

$$
\begin{aligned}
& \bar{\phi}^{(1)}=\bar{\Phi}_{1}^{(1)}=C_{1} \cosh (|\xi| Z)+\frac{\mathrm{i} \xi B_{1}}{2|\xi|} Z \sinh (|\xi| Z), \quad 0<Z<1, \\
& \bar{\phi}^{(1)}=\bar{\Phi}_{2}^{(1)}=C_{2} \exp (-|\xi| Z)-\frac{\mathrm{i} \xi B_{2}}{2|\xi|} Z \exp (-|\xi| Z), \quad Z>1,
\end{aligned}
$$

where $B_{1}$ and $B_{2}$ have been determined as part of the solution for $\Phi^{(0)}$. On $Z=1, \mathrm{~d} \Phi^{(1)} / \mathrm{d} Z$ is continuous, with

$$
\begin{array}{rlrl}
\frac{\partial \Phi^{(1)}}{\partial Z} & =\text { unknown, } & & X>0 \\
& =0 & X<0
\end{array}
$$

and $\bar{\Phi}^{(1)}$ must also satisfy the condition

$$
\frac{\partial \bar{\Phi}^{(1)}}{\partial Z}=0, \quad \text { on } Z=0, \quad \forall X .
$$

Finally, after some algebra we deduce the functional equation relating $W_{+}^{(1)}$ to $U_{-}^{(1)}=\bar{\Phi}_{1}^{(1)}\left(\xi, 1^{-}\right)-$ $\bar{\Phi}_{1}^{(1)}\left(\xi, 1^{+}\right)$, corresponding to the jump in transformed potentials on $Z=1$ :

$$
\frac{W_{+}^{(1)}}{G_{+}}-\frac{\hat{A} \mathrm{i}}{|\xi|[\exp (2|\xi|)-1]}=G_{-} U_{1}^{(1)}+\frac{\mathrm{i} \hat{A}}{2 \xi^{2}}
$$

where $G=G_{+} G_{-}$is defined in equations (4.14)-(4.16).

We now need the sum split of the second term on the left-hand side of (4.35). To do this, we write

$$
\frac{1}{|\xi|\left(\mathrm{e}^{2|\xi|}-1\right)}=\left[\frac{1}{|\xi|\left(\mathrm{e}^{2|\xi|}-1\right)}-\frac{\mathrm{e}^{-|\xi|}}{2 \xi^{2}}\right]+\frac{\mathrm{e}^{-|\xi|}}{2 \xi^{2}}=F_{1}(\xi)+\frac{F_{2}(\xi)}{2 \xi^{2}}
$$

and each of the $F_{i}$ can be written as a sum of plus and minus functions using Cauchy's theorem. Thus

$$
F_{1 \pm}= \pm \frac{1}{2 \pi \mathrm{i}} \int_{-\infty}^{\infty} \frac{F_{1}(\xi)}{\xi-z} \mathrm{~d} \xi
$$

where the contour for $F_{+}$lies just below the real axis and that for $F_{-}$lies just above the axis. We multiply the integrand top and bottom by $(\xi+z)$ to obtain

$$
\begin{aligned}
F_{1 \pm}(z) & = \pm \frac{1}{2 \pi \mathrm{i}} \int_{-\infty}^{\infty}\left[\frac{1}{|\xi|\left(\mathrm{e}^{2|\xi|}-1\right)}-\frac{\mathrm{e}^{-|\xi|}}{2 \xi^{2}}\right] \frac{(\xi+z)}{\xi^{2}-z^{2}} \mathrm{~d} \xi \\
& = \pm \frac{1}{\pi \mathrm{i}} \int_{0}^{\infty}\left[\frac{1}{x\left(\mathrm{e}^{2 x}-1\right)}-\frac{\mathrm{e}^{-x}}{2 x^{2}}\right] \frac{z \mathrm{~d} x}{x^{2}-z^{2}},
\end{aligned}
$$

where the term that is antisymmetric in $x$ has been eliminated from the second expression. Note that the integrand in (4.38) is finite at $x=0$, and for sufficiently small $x$ the term in square brackets can be approximated as $(-1+x+\cdots) / 12$, and hence

$$
F_{1-}(0)=F_{1+}(0)=-\frac{1}{6}
$$


Similarly, for $F_{2}(z)=\exp (-|z|)$,

$$
F_{2 \pm}(z)= \pm \frac{1}{2 \pi \mathrm{i}} \int_{-\infty}^{\infty} \frac{\mathrm{e}^{-|\xi|}}{\xi-z} \mathrm{~d} \xi= \pm \frac{1}{\pi \mathrm{i}} \int_{0}^{\infty} \frac{z \mathrm{e}^{-x} \mathrm{~d} x}{x^{2}-z^{2}}
$$

Thus (4.36) is sum-split as

$$
F_{1}(\xi)+\frac{F_{2}(\xi)}{2 \xi^{2}}=\left\{F_{1+}(\xi)+\frac{F_{2+}(\xi)}{2 \xi^{2}}\right\}+\left\{F_{1-}(\xi)+\frac{F_{2-}(\xi)}{2 \xi^{2}}\right\} .
$$

As $|z| \rightarrow \infty$, in the respective half planes

$$
\begin{aligned}
& F_{1 \pm}(z) \sim \pm \frac{1}{\pi \mathrm{i}}\left(\frac{1}{z}\right) 0.0723649+O\left(z^{-3}\right), \\
& F_{2 \pm}(z) \sim \pm \frac{1}{\pi \mathrm{i}}\left(-\frac{1}{z}\right),
\end{aligned}
$$

where (4.42a) was obtained from (4.38) by means of the quadgk routine within Matlab [21]. Re-arranging (4.35), we have

$$
\frac{W_{+}^{(1)}}{G_{+}}-\mathrm{i} \hat{A} F_{1+}-\mathrm{i} \hat{A} \frac{F_{2+}}{2 \xi^{2}}=G_{-}^{(1)} U_{-}^{(1)}+\frac{\mathrm{i} \hat{A}}{2 \xi^{2}}+\mathrm{i} \hat{A} F_{1-}+\mathrm{i} \hat{A} \frac{F_{2-}}{2 \xi^{2}}=\frac{\hat{B}_{1}}{\xi}+\frac{\hat{B}_{2}}{\xi^{2}},
$$

where the asymptotic behaviour of the \pm functions as $\xi \rightarrow \infty$ can be determined in the same manner as at zeroth order (and using also (4.42)). Equality of the + and - functions is then ensured by analytic continuation, since they are equal along the real axis.

\subsection{The inner solution in the limit $X \rightarrow-\infty$}

The terms $\hat{B}_{1} \xi^{-1}+\hat{B}_{2} \xi^{-2}$ in (4.43) represent eigensolutions of the inner problem to match with the outer solution. Matching as $X \rightarrow-\infty$ in real space requires expansions of $U_{-}, G_{-}, F_{1-}$ and $F_{2-}$ as $\xi \rightarrow 0$ in Fourier space.

$F_{1-}(\mathrm{i} \eta)$, given by (4.38), tends to a constant as $\eta \rightarrow 0_{+}$. To expand $F_{2-}(z)(4.40)$ as $z \rightarrow 0$, we set $z=-\mathrm{i} \eta$ and let $\eta \rightarrow 0+$, so that we are in the lower half plane. Then

$$
F_{2-}(-\mathrm{i} \eta)=\frac{\eta}{\pi} \int_{0}^{\infty} \frac{\mathrm{e}^{-x} \mathrm{~d} x}{x^{2}+\eta^{2}} \sim \frac{1}{\pi}\left[\frac{\pi}{2}+\eta(\gamma-1)+\eta \ln \eta-\frac{\pi}{4} \eta^{2}+\cdots\right], \quad \eta \rightarrow 0_{+} .
$$

From Eqns (4.43) and (4.44) (with $\eta=\mathrm{i} \xi$ ) we have

$$
\begin{aligned}
U_{-}^{(1)} & =\frac{1}{G_{-}}\left\{\frac{\hat{B}_{1}}{\xi}+\frac{\hat{B}_{2}}{\xi^{2}}-\frac{\mathrm{i} \hat{A}}{2 \xi^{2}}-\mathrm{i} \hat{A} F_{1-}(0)-\frac{\mathrm{i} \hat{A}}{2 \xi^{2}} F_{2-}(\xi)\right\} \\
& =\frac{1}{G_{-}}\left\{\frac{1}{\xi^{2}}\left(\hat{B}_{2}-\frac{3 \mathrm{i}}{4} \hat{A}\right)-\frac{\hat{A}}{2 \xi \pi}[(1-\gamma)-\ln (\mathrm{i} \xi)]+\frac{\hat{B}_{1}}{\xi}+\cdots\right\} .
\end{aligned}
$$

Hence, combining (4.24) and (4.45), we obtain

$$
\begin{aligned}
U_{-}^{(1)} \sim & \frac{1}{\xi^{3}}\left(\hat{B}_{2}-\frac{3 \mathrm{i}}{4} \hat{A}\right)+\frac{1}{\pi \xi^{2}}[(1-\gamma)-\ln (\mathrm{i} \xi)]\left(\mathrm{i} \hat{B}_{2}+\frac{3}{4} \hat{A}-\frac{\hat{A}}{2}\right) \\
& +\frac{\hat{B}_{1}}{\xi^{2}}+\frac{\mathrm{i}}{\pi \xi^{2}} \ln \pi\left(\hat{B}_{2}-\frac{3 \mathrm{i}}{4} \hat{A}\right)+\cdots .
\end{aligned}
$$


We invert (4.46) by means of (4.26a), and obtain the contribution to the inner solution (in the limit $X \rightarrow-\infty)$

$$
\begin{aligned}
{\left[V^{(1)}\right] } & =V_{h^{+}}^{(1)}-V_{h^{-}}^{(1)} \\
& =\frac{\mathrm{i} X^{2}}{2}\left(\hat{B}_{2}-\frac{3 \mathrm{i}}{4} \hat{A}\right)-\frac{X \ln (|X|)}{\pi}\left(\mathrm{i} \hat{B}_{2}+\frac{\hat{A}}{4}\right)-\left[\hat{B}_{1}+\frac{\mathrm{i}}{\pi} \ln \pi\left(\hat{B}_{2}-\frac{3 \mathrm{i}}{4} \hat{A}\right)\right] X+\cdots .
\end{aligned}
$$

Adding (4.27) and (4.47), we find the outer expansion of the inner solution

$$
\begin{aligned}
{[V] } & =\left[V^{(0)}\right]+h\left[V^{(1)}\right]=-\hat{A}\left\{X-\frac{1+\ln \pi}{\pi}-\frac{\ln (-X)}{\pi}\right\}+\frac{\mathrm{i} h X^{2}}{2}\left(\hat{B}_{2}-\frac{3 \mathrm{i}}{4} \hat{A}\right) \\
& -\frac{h X \ln (|X|)}{\pi}\left(\mathrm{i} \hat{B}_{2}+\frac{\hat{A}}{4}\right)-\left[\hat{B}_{1}+\frac{\mathrm{i}}{\pi} \ln \pi\left(\hat{B}_{2}-\frac{3 \mathrm{i}}{4} \hat{A}\right)\right] h X+\cdots, \quad \text { as } X \rightarrow \infty .
\end{aligned}
$$

\subsection{The inner solution in the limit $X \rightarrow 0$}

To find the behaviour of the inner solution as $X \rightarrow 0$ we follow the same procedure as at zeroth order. From (4.43) and (4.42a), we see that

$$
G_{-}^{(1)} U_{-}^{(1)} \sim-\mathrm{i} \hat{A} F_{1-}+\frac{\hat{B}_{1}}{\xi}+\frac{\hat{B}_{2}}{\xi^{2}}-\frac{\mathrm{i} \hat{A}}{2 \xi^{2}}
$$

and hence, using (4.42a) and (4.18),

$$
U_{-}^{(1)} \sim \frac{(2 \mathrm{i})^{1 / 2}}{\xi^{3 / 2}}\left(0.0723649 \frac{\hat{A}}{\pi}+\hat{B}_{1}\right)+\frac{(2 \mathrm{i})^{1 / 2}}{\xi^{5 / 2}}\left[\hat{B}_{2}-\frac{B_{1} \pi \mathrm{i}}{12}-\mathrm{i} \hat{A}\left(\frac{1}{2}+\frac{0.0723649}{12}\right)\right]
$$

Inversion of (4.50) by means of (4.28) yields

$$
\begin{aligned}
{\left[V^{(1)}\right] } & =V_{h^{+}}^{(1)}-V_{h^{-}}^{(1)}=\left(0.0723649 \frac{\hat{A}}{\pi}+\hat{B}_{1}\right) 2\left(\frac{2}{\pi}\right)^{1 / 2}(-X)^{1 / 2} \\
& +h\left[\mathrm{i} \hat{B}_{2}+\frac{B_{1} \pi}{12}+\hat{A}\left(\frac{1}{2}+\frac{0.0723649}{12}\right)\right] \frac{4}{3}\left(\frac{2}{\pi}\right)^{1 / 2}(-X)^{3 / 2} \text { as } X \rightarrow 0 .
\end{aligned}
$$

\section{Matching}

In our matching procedure it will occur naturally that we should consider terms of order $h$ and order $h \ln h$ as being of the same order. For example, in equation (5.4) below, we treat $A$ as a constant which includes a term $\ln h$ which changes only slowly (e.g. from -9.21034 to twice that as $h$ decreases from $10^{-4}$ to $10^{-8}$ ).

The outer solution for jump in potential across the disks is given by combining (3.29) and 
(3.32):

$$
\begin{aligned}
V_{h^{+}}-V_{h^{-}} & =V_{h^{+}}^{(0)}-V_{h^{-}}^{(0)}+h\left[V_{h^{+}}^{(1)}-V_{h^{-}}^{(1)}\right] \\
& =\frac{r^{2}-1}{4 h}+A-\int_{r_{1}}^{1} \frac{\left(1-2 t_{1}\right)}{2 \pi^{2} t_{1}^{1 / 2}\left(1-t_{1}\right)^{1 / 2}\left(t_{1}-r_{1}\right)^{1 / 2}} \ln \left[\frac{1+t_{1}^{1 / 2}}{1-t_{1}^{1 / 2}}\right] \mathrm{d} t_{1},
\end{aligned}
$$

which can be expanded in the limit $h=(r-1) / X \ll 1$ as

$$
\begin{aligned}
V_{h^{+}}-V_{h^{-}} & =\frac{X}{2}+\frac{h X^{2}}{4}+A+\frac{3}{2 \pi} \ln 2-\frac{\ln h}{2 \pi}-\frac{\ln (-X)}{2 \pi} \\
& +\frac{h X}{4 \pi}[9 \ln 2-3-3 \ln h]-\frac{3}{4 \pi} X h \ln (-X)+O\left(h^{2} X^{2}\right) .
\end{aligned}
$$

We compare (5.2) with the outer expansion (4.48) of the inner solution in order to match the inner solution to the outer solution. Comparing first the terms in $X$ we conclude that

$$
\hat{A}=-\frac{1}{2} \text {. }
$$

The term $\hat{A} \ln (-X) / \pi$ in the zeroth order inner expansion (4.48) immediately matches with the corresponding term in the outer expansion (5.2). Matching the constant terms in (5.2) and (4.48) implies that

$$
A+\frac{3 \ln 2}{2 \pi}-\frac{\ln h}{2 \pi}=-\frac{1+\ln \pi}{2 \pi},
$$

and hence

$$
A=-\frac{1}{2 \pi}(1+\ln \pi+3 \ln 2-\ln h) .
$$

Terms $O\left(h X^{2}\right)$ give

$$
\hat{B}_{2}=-\frac{7 \mathrm{i}}{8}
$$

This choice of $\hat{B}_{2}$ also ensures that terms $O(h X \ln X)$ match. Matching terms $O(h X)$ gives

$$
\hat{B}_{1}+\frac{\mathrm{i}}{\pi} \ln \pi\left(\hat{B}_{2}-\frac{3 \mathrm{i}}{4} \hat{A}\right)=-\frac{1}{4 \pi}(9 \ln 2-3-3 \ln h),
$$

and hence

$$
\hat{B}_{1}=\frac{1}{4 \pi}[3-9 \ln 2+3 \ln h-2 \ln \pi] .
$$

Now that $\hat{A}$ and $\hat{B}_{1}$ are known, the inner expansion in the limit $X \rightarrow 0$ can be expressed by (4.30) and (4.51) as

$$
\begin{aligned}
{[V]=V_{h^{+}} } & -V_{h^{-}}=-(-X)^{1 / 2}\left(\frac{2}{\pi}\right)^{1 / 2}[1+h(0.90281-0.47746 \ln (h))+\cdots] \\
& +(-X)^{3 / 2}[-0.139257+0.5392 h+0.06649 h \ln h]+\cdots, \quad \text { as } X \rightarrow 0 .
\end{aligned}
$$

Figure 2 shows the inner and outer solutions for $[V]$ near $X=0$ for the cases $h=0.1$ and $h=0.01$. 


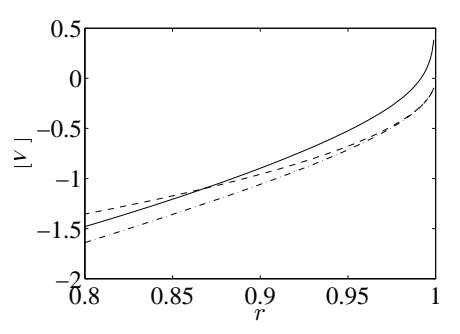

(a)

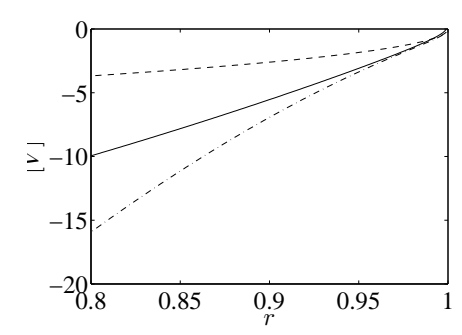

(b)

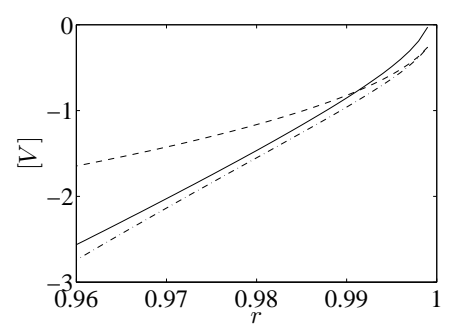

(c)

FIgURE 2. The jump $[V]=V_{h^{+}}-V_{h^{-}}$as a function of $r \longrightarrow$ Two-term expansion (5.1) of the outer solution; - - - Two-term expansion (5.9) of inner solution to $O\left((-X)^{1 / 2}\right)$; - - - - Two-term expansion (5.9) of inner solution, to $O\left((-X)^{3 / 2}\right)$. (a) $h=0.1$, (b,c) $h=0.01$, (c) is a detail of (b).

\section{Conclusion: the added mass}

To determine the added mass we first form the composite expansion

$$
\phi^{(\mathrm{comp})}(r, z)=V(r, z)+\phi^{(\text {in })}-\phi^{(\text {overlap })}
$$

where $V(r, z)=V^{(0)}+h V^{(1)}+O(h)$ is the two-term outer solution (5.1), $\phi^{(\text {in) }}$ is the two term inner solution (4.2) and $\phi^{(\text {overlap) }}$ is the outer expansion (4.48) of the inner solution.

The added mass of the disk is given by (2.8) and (3.27):

$$
\begin{aligned}
M & =-2 \pi \int_{r=0}^{1} r\left[\phi\left(r, h^{+}\right)-\phi\left(r, h^{-}\right)\right] \mathrm{d} r \\
& =-2 \pi \int_{r=0}^{1} r\left[\phi^{(\mathrm{comp})}\left(r, h^{+}\right)-\phi^{(\mathrm{comp})}\left(r, h^{-}\right)\right] \mathrm{d} r+O(h) .
\end{aligned}
$$

We do not have a simple analytic expression for the inner solution over the full range of $X$, but we know its Fourier transform. Hence we know that $\phi^{(\text {in) }}-\phi^{\text {(overlap) }} \rightarrow 0$ as $X \rightarrow-\infty$, and that $\phi^{(\text {in) }}$ and $\phi^{\text {(overlap) }}$ are at most $O(1)$ if $X$ is $O(1)$ (i.e. if $1-r$ is $O(h)$ ), so that the contribution of $\phi^{\text {(in) }}-\phi^{\text {(overlap) }}$ to the integral $(6.2 b)$ is $O(h)$. Our aim here is to determine the added mass to $O(1)$, and we can therefore approximate the jump in potential $\phi$ in the integral $(6.2 a)$ by the jump (5.1) of the outer solution alone. The integral of jump in the zeroth order term of the outer solution (3.29) is straightforward, and the first order contribution (3.32) can be integrated by interchanging the order of integration with respect to $r$ and $t_{1}$. We find

$$
M=\frac{\pi}{8 h}-A \pi-\frac{4}{3}+\cdots=\frac{\pi}{8 h}-\frac{\ln h}{2}+0.77875 .
$$

The leading order term $\pi /(8 h)$ in the expansion for $M$ can be obtained by means of an anaysis of inviscid flow in the gap between the disks, ignoring fluid outside the gap [12]. The numerical results of Sherwood [12] are shown as the open triangles in figure 3a. We see that the analytic prediction (6.3) appears to be a good fit to the numerical results. However, we also see that the leading order term $\pi /(8 h)$ dominates the results as $h \rightarrow 0$. A more stringent test is to make comparisons after this leading order term has been subtracted from all results. Such comparisons are shown in figure $3 \mathrm{~b}$. We might expect that the errors in the analytic asymptote (6.3) are $O(h \ln h)$ and decay slowly as $h \rightarrow 0$, so that the difference between the numerical results and analytic prediction in figure $3 \mathrm{~b}$ is acceptable. However, it would seem that the attempt 


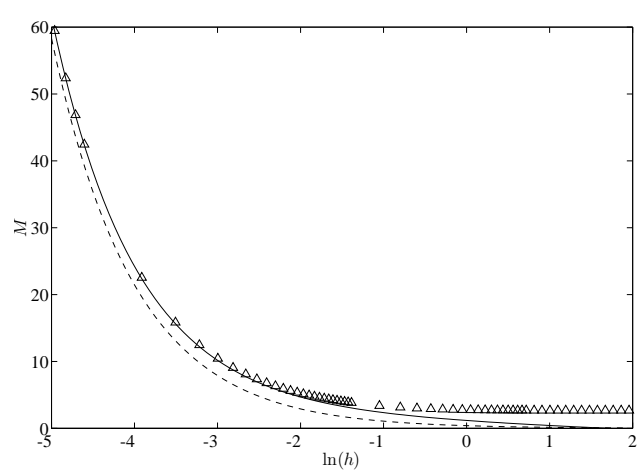

(a)

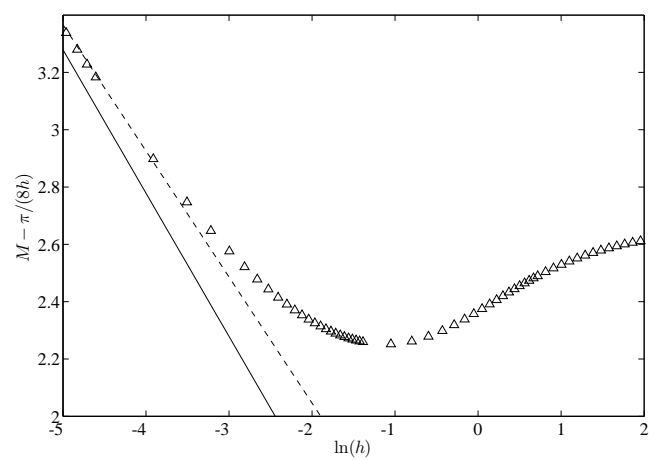

(b)

FIGURE 3. (a) Added mass $M$ of one disk as a function of half gap-width $h . \triangle$ numerical results from [12]; - - — — leading order asymptote $M=\pi /(8 h)$; — analytic prediction (6.3). (b) Difference $M-\pi /(8 h)$ between the added mass $M$ and the leading order asymptote $\pi /(8 h)$, as a function of half gap-width $h . \triangle$ numerical results from [12]; — analytic prediction (6.3); - — — - fit (6.4) to numerical results.

in [12] to fit the numerical results to a curve of the form

$$
M=\frac{\pi}{8 h}-0.44 \ln h+1.166
$$

was not sufficiently accurate to permit extrapolation to smaller values of $h$ than those for which computations were performed (see figure $3 \mathrm{~b}$ ).

\section{Acknowledgement}

JDS thanks the Department of Applied Mathematics \& Theoretical Physics, University of Cambridge, for hospitality.

\section{References}

[1] Maxwell, J. C. (1866) The Bakerian lecture: On the viscosity or internal friction of air and other gases. Phil. Trans R. Soc. 156, 249-268.

[2] Kirchioff, G. R. (1877) Zur Theorie des Condensators. Monatsberichte der Königlich Preussischen Akademie der Wissenschaften zu Berlin. 144-162.

[3] Cooke, J. C. (1958) The coaxial circular disc problem. Z. Angew. math. und Mech. 38, 349-356.

[4] Hutson, V. (1963) The circular plate condenser at small separations. Proc. Camb. Philos. Soc. 59, 211-224.

[5] Sneddon, I. N. (1966) Mixed boundary value problems in potential theory. Amsterdam. NorthHolland.

[6] Shaw, S. J. N. (1970) Circular-disc viscometer and related electrostatic problems. Phys. Fluids 13, 1935-1947.

[7] AtKinson, C. \& Leppington, F. G. (1983) The asymptotic solution of some integral equations. IMA J. Applied Math. 31, 169-182.

[8] Lawrence, C. J., Kuang, Y. \& Weinbaum S. (1985) The inertial draining of a thin fluid layer between parallel plates with a constant normal force. Part 2. Boundary layer and exact numerical solutions. J. Fluid Mech. 156, 479-494. 
[9] Moss, E. A., Krassnokutski, A., Skews, B. W. \& Paton R. T. (2011) Highly transient squeeze-film flows. J. Fluid Mech. 671, 384-398.

[10] Brosse, N. \& ERn, P. (2011) Paths of stable configurations resulting from the interaction of two disks falling in tandem. J. Fluids Structures 27, 817-823.

[11] Brosse, N. \& ERn, P. (2014) Interaction of two axisymmetric bodies falling in tandem at moderate Reynolds numbers. J. Fluid Mech. 757, 208-230.

[12] Sherwood, J. D. (2011) Added mass of a pair of discs. Phys. Fluids 23, 103601.

[13] TRANTER, C. J. (1956) Integral transforms in mathematical physics. London. Methuen.

[14] Tranter, C. J. (1968) Bessel functions with some physical applications. London. The English Universities Press.

[15] Chen, C. Y. \& AtKinson, C. (1997) The axisymmetric problem of a disc in a bimaterial sandwich - (the history dependent) scalar potential case. Int. J. Engng Sci. 35, 229-251.

[16] MARTIN, P. A. \& FARINA, L. (1997) Radiation of water waves by a heaving submerged horizontal disk. J. Fluid Mech. 337, 365-379.

[17] FARINA, L. (2010) Water wave radiation by a heaving submerged horizontal disk very near the free surface. Phys. Fluids 22, 057102.

[18] Love, E. R. (1949) The electrostatic field of two equal circular co-axial conducting disks. $Q$. J. Mech. Appl. Math. 2, 428-451.

[19] Batchelor, G. K. (1973) An introduction to fluid dynamics. Cambridge. Cambridge University Press.

[20] Collins, W. D. (1959) On the solution of some axisymmetric boundary value problems by means of integral equations, II: further problems for a circular disc and a spherical cap. Mathematika $\mathbf{6}$, $120-133$.

[21] MATLAB Release 2014a, The MathWorks, Inc., Natick, Massachusetts, United States. 